\title{
Myelin loss may explain how autism- linked DNA deletion affects cognition
}

\section{BY ANNA GOSHUA}

8 DECEMBER 2021

Mice that lack one copy of TBX1, a gene in the autism-linked 22q11.2 chromosomal region, produce too little myelin - the fatty insulation that surrounds neurons - and perform poorly on tasks that measure cognitive speed, according to a new study.

The work, published 5 November in Molecular Psychiatry, may offer insight into the mechanisms that underlie impaired cognitive function in some people with a 22q11.2 deletion, and possibly other copy number variants (CNVs).

"The myelin changes could potentially emerge as a common neuronal deficit that mediates cognitive changes among many CNV cases," says lead investigator Noboru Hiroi, professor of pharmacology at the University of Texas Health Science Center at San Antonio.

Neuronal axons - the projections that conduct nerve impulses — are coated with myelin, which serves to speed up electrical transmission. The brains of autistic people and several mouse models of autism have disruptions in myelin, previous research has shown.

These connecting fibers are the "highways of the brain," says Valerie Bolivar, research scientist at the New York State Department of Health's Wadsworth Center in Albany. "If the highway doesn't work, you can't get your goods from one place to another as fast."

\section{Slow mice:}

TBX1 encodes a protein that regulates the expression of other genes during brain development. Deleting one copy of TBX1 leads to social and communication deficits in mice, according to previous studies by Hiroi's team.

In the new study, the researchers imaged the brains of the TBX1 mice and found significant myelin 


\section{Spectrum | Autism Research News}

https://www.spectrumnews.org

alterations in only the fimbria, a bundle of nerve fibers within the hippocampus - a critical structure for learning and memory. Large and medium axons in the fimbria had unusually low amounts of myelin in the mutated mice, microscopy analyses showed.

This brain region showed decreased expression of NG2, a gene required for the brain to produce the precursors of oligodendrocytes, the cells that make myelin. Another zone of the fimbria also produced fewer oligodendrocytes.

The TBX1 mice faltered on several behavioral tests. They were worse than their wildtype littermates at navigating their surroundings in the Morris water maze, which tests spatial memory and relies on an intact hippocampus. And they performed more slowly on an attentional set-shifting task, which involves shifting attention from one stimulus to another, to measure attention and cognitive flexibility.

"When myelin is affected, that can influence how efficiently the entire brain operates," says Carrie Bearden, professor of psychiatry and biobehavioral sciences at the University of California, Los Angeles, who was not involved in the study. "In humans, what it really tends to affect is processing speed."

Understanding how CNVs may influence specific cognitive functions is key for developing treatments.

"We have the starting point for precision medicine. You can try targeting myelin in the fimbria specifically rather than the whole brain," Hiroi says.

But researchers caution that the observed myelination changes in mice don't necessarily correspond to what is going on in the human brain and that the findings require validation in people.

"The degree of myelination in a mouse brain is completely different from humans," Bearden says.

Moving forward, the researchers plan to investigate whether their findings are generalizable to other CNVs.

Cite this article: https://doi.org/10.53053/ZFYM6324 